\title{
Structural and electronic properties of $\beta-\mathrm{MnO}_{2}$ employing DFTB technique
}

\author{
P Ngobeni, PE Ngoepe, KP Maenetja \\ Materials Modelling Centre, University of Limpopo, Private Bag X1106, 0727, Sovenga, South Africa \\ Email:percy.ngobeni@ul.ac.za
}

\begin{abstract}
$\mathrm{MnO}_{2}$ is presently under massive review for its capacitance properties. $\mathrm{MnO}_{2}$ recrystallizes into several crystallographic structures such as $\alpha, \beta, \gamma, \delta$, and $\lambda$ structure. These structures vary in the way $\mathrm{MnO}_{6}$ octahedra are connected, they possess tunnels or interlayers with gaps of different magnitudes. However, upon lithium intercalation in $\beta-\mathrm{MnO}_{2}, \mathrm{LiMnO}_{2}$ suffers from capacity loss due to undesirable structural phase transformation into spinel like $\operatorname{LixMn}_{2} \mathrm{O}_{4}$. One of the major demands is to modify and strengthen the structural stability of $\mathrm{MnO}_{2}$ to prevent phase transformation during lithium intercalation and rapid capacity fading during cycling. DMol ${ }^{3}$ is a density functional theory-based program used to calculate the lattice parameter of ferromagnetic $\mathrm{MnO}_{2}$. After successfully parameterized $\mathrm{MnO}_{2}$, the lattice parameters were compared with the results from experiments. Density functional tight-binding (DFTB) was employed to investigate the electronic properties of $\mathrm{MnO}_{2}$ such as density of states (DOS) and band structures. The DOS was calculated to check the conductivity of $\mathrm{MnO}_{2}$. The electronic band structures calculated indicate the absence of a gap at the Fermi level, thus $\mathrm{MnO}_{2}$ is metallic. These findings are important in preserving the crystal structure of $\mathrm{LiMnO}_{2}$ and the maintenance of capacity during cycling.
\end{abstract}

Keywords: Capacity, Stability, Parameters, Fermi level

\section{Introduction}

Manganese dioxide is a material that is most promising in terms of electrode materials concerning it is easy preparation, low price, and toxicity (Thackeray et al., 1983). They provide high energy however power density remains too low for high electricity applications. A challenge is to put into effect battery technology in large-scale excessive-strength structures, while a growing market for Li-ion batteries in electric powered automobiles and hybrid vehicles requires $\mathrm{Li}$-ion batteries with not only high power, excessive capability, excessive charging rate, and lengthy life but additionally improved safety overall performance. It has been broadly applied for commercial primary lithium batteries since the mid-70s (Hunter, 1981). These manganese dioxide materials not only improve the lithium-ion batteries, however acquired interest from various fields consisting of catalysis, and electrochemistry. The $\mathrm{MnO}_{2}$ nanostructure is regarded because the promising electrocatalysts concerning the OER in alkaline solutions due to its excessive catalytic activity and outstanding stability of resistance. However, upon lithium intercalation in $\beta-\mathrm{MnO}_{2}, \mathrm{LiMnO}_{2}$ suffers from capacity loss due to undesirable structural phase transformation into spinel like $\mathrm{LixMn}_{2} \mathrm{O}_{4}$. Some researchers are concerned that (1x1) tunnels (Turner et al.,1981) of $\beta-\mathrm{MnO}_{2}$ can be too narrow to deal with sufficient Li-ions at room temperature. research (Thackeray et al., 1989), confirmed that the amount of lithium chemically inserted into crystallized $\beta-\mathrm{MnO}_{2}$ changed into less than $\mathrm{Li} / \mathrm{Mn}=0.3$, it reaches close to $\mathrm{Li} / \mathrm{Mn}=1$ most effective at temperatures above $50{ }^{\circ} \mathrm{C}$ or in poorly crystallized products (Thackeray et al.,1989;Zachau-Christiansen et al., 1994).
Table 1: The different polymorphs of $\mathrm{MnO}_{2}$ and their tunnels (Dahn et al.,1991)

\begin{tabular}{lll}
\hline $\mathbf{M n O}$ type & Structure type & $\begin{array}{l}\text { Interstitial space of the } \\
\mathbf{M n O}_{2}\end{array}$ \\
\hline$\alpha-\mathrm{MnO}_{2}$ & Hollandite & $(1 \times 1)$ and $(2 \times 2)$ tunnels \\
$\beta-\mathrm{MnO}_{2}$ & Pyrolusite & $(1 \times 1)$ tunnels \\
$\lambda-\mathrm{MnO}_{2}$ & Spinel & $\begin{array}{l}\text { 3-dimensional network of } \\
\text { tunnels }\end{array}$ \\
$\gamma-\mathrm{MnO}_{2}$ & $\begin{array}{l}\text { Intergrowth of } \\
\text { pyrolusite and }\end{array}$ & $(1 \times 1)$ and $(1 \times 2)$ tunnels \\
$\delta-\mathrm{MnO}_{2}$ & $\begin{array}{l}\text { Ramsdellite } \\
\text { Layered (hydrated })\end{array}$ & $\begin{array}{l}\text { Layers of } \mathrm{H}_{2} \mathrm{O} \text { and } \\
\text { stabilizing cations }\end{array}$ \\
\hline
\end{tabular}

Pyrolusite also identified as $\beta-\mathrm{MnO}_{2}$, is the stable form of $\mathrm{MnO}_{2}$ at ambient conditions and has the tetragonal rutile structure (Kim et al., 2006). The $\mathrm{MnO}_{2}$ system exists in distinctive sorts of polymorphs which might be primarily based on the assembly of the octahedral $\mathrm{MnO}_{6}$ subunits shared through corners or edges. The variation of the shape kind with interstitial space of the $\mathrm{MnO}_{2}$ system is observed in table 1 above. some of the manganese dioxide-primarily based material properties that make this system valuable are the distinct crystal shape and oxidation states.

We improve structural stability and capacity loss during cycling during lithium intercalation in $\beta-\mathrm{MnO}_{2}$ by investigating the structural and electronic properties of $\beta-\mathrm{MnO}_{2}$ using the DFTB technique and comparing the results with experimental and DFT work. The development of lattice parameters for $\beta-\mathrm{MnO}_{2}$ gives better insight information of the structures. 


\section{Methodology}

Density functional tight-binding (DFTB) calculations were performed within the generalized gradient approximation (GGA) in the form of a PBE exchange-correlation function coupled with a DND basis set (Perdew et al.,1996). Atomic parameters for the interaction between atoms in DFTB + are required to perform various calculations such as geometry optimization. $\mathrm{DMol}^{3}$ is a density functional theory program that predicts the properties of various materials, such as structural and electronic properties (Delley.,1990). The number of plan waves was determined by a kinetic energy cut-off of $500 \mathrm{eV}$. The number of k-points was determined by performing single-point calculations using $\mathrm{DMol}^{3}$, these k-points have been determined such that the total energy difference per atom is equal or less than $1 \mathrm{meV}$, as this is a wellknown acceptance criterion for proper convergence in DFT calculations. The k-points mesh $6 \times 6 \times 9$ were found to be sufficient to converge the $\beta-\mathrm{MnO}_{2}$ system.

The $\mathrm{DMol}^{3}$ was used for geometry optimization of $\beta-\mathrm{MnO}_{2}$, which was maintained by all the interactions of $\beta-\mathrm{MnO}_{2}$ such as $\mathrm{Mn}-\mathrm{Mn}, \mathrm{Mn}-\mathrm{O}, \mathrm{O}-\mathrm{Mn}$, and O-O. These structures were prepared to be at their ground state energy before being used for the selfconsistent-charge density functional tight-binding (SCC-DFTB) parameterization process. $\mathrm{Dmol}^{3}$ allowed the modelling of the electronic structures and energetics of molecules, solids, and surfaces using density functional theory (DFT), which produced accurate results before starting with parameterization.

The $\beta-\mathrm{MnO}_{2}$ bulk structure is a primitive tetragonal with space group $\mathrm{P} 4_{2} / \mathrm{mnm}$ (Mo et al.,1995), it is characterized by an octahedrally coordinated $\mathrm{Mn}$ atom. The building blocks of the $\beta-\mathrm{MnO}_{2}$ system are the columns of edge-sharing $\mathrm{MnO}_{6}$ octahedra, these columns be a part of either corner-to-corner or edge-to-edge (Cockayne et al.,2012). The pyrolusite structures may be defined as a finite single chain, while the ramsdellite structures have dual chains (MacLean et al.,1996). Pyrolusite is identical to that of rutile $\mathrm{TiO}_{2}$, the oxygen atoms have primitive tetragonal packing (West.,1983).

\section{Results and discussion}

\section{Structural and properties of $\beta-\mathrm{MnO}_{2}$}

A brief discussion of how the DFTB + parameters are developed and compared to experimental and DFT results. The potentials used

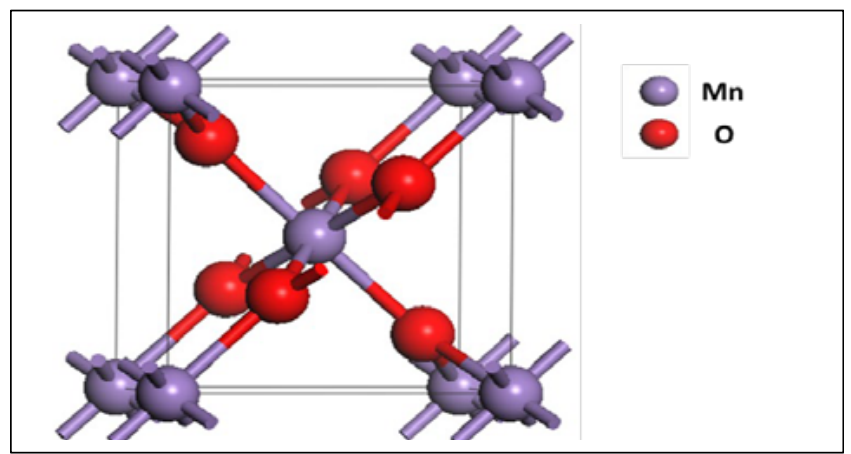

Figure 1: Pyrolusite unit cell of $\mathrm{MnO} 2$ with purple spheres representing the metal atom $\mathrm{Mn}$ and red spheres representing the oxygen atom were derived using DMol3 and SCC-DFTB. Before the emerging these parameters, several considerations when parameterizing the reference systems for $\mathrm{MnO}_{2}$ such as DFT and DFTB calculations, the fitting process, Slater-Koster library file generation, data coverage, degrees of freedom, and the number of trials, and elementpair coverage. To fit repulsion functions for the Mn-Mn, Mn-O, and $\mathrm{O}-\mathrm{O}$. We used a fit set involving a manganese dioxide system (with very low weight). The molecular fit paths were created by perturbing and scaling the manganese and oxygen atoms presented as the reference structures. We were able to generate a set of Mn$\mathrm{Mn}, \mathrm{Mn}-\mathrm{O}$, and O-O repulsive potentials which mimic energy and geometrical data in the identical quality as the reference handmade set. The parametrization was achieved by adjusting the wave function confinement radius (Radii 1) and density confinement radius (Radii 2) for $\mathrm{MnO}$ elements. The parameters are developed successfully when Radii 1 was set to $\mathrm{Mn}=2.6$ and $\mathrm{O}=2.1 \mathrm{Bohr}$ and Radii 2 set to $\mathrm{Mn}=6.0$ and $\mathrm{O}=6.0$.

The lattice parameter obtained from full geometry optimization with DTFB + code are recorded below (Table 2) and some available experimental and DFT data. The attained values of the equilibrium lattice parameters from DFTB + code together with experimental data and DFT data were compared. Our results below were found to be in good agreement with the others that are obtained by other researchers using various methods. The obtained lattice parameters are within $2 \%$ of the experimental data.

\section{Electronic properties of $\beta-\mathrm{MnO}_{2}$}

The electronic properties of materials help with the insights through the categorization of materials under three main phases such as semiconductors, metal, and insulators. The description of a material is determined by the size and existence of the energy gap between the highest occupied orbitals (conduction band) and the lowest unoccupied orbitals (valence band). In semiconductors and insulators, the gap is present, which happens to be larger for insulators. In metals, the occupied and unoccupied orbitals overlap, hence no gap between the bands is observed. High DOS at precise energy approach that there are numerous states available for

Table 2: Lattice parameters of $\beta-\mathrm{MnO}_{2}$ obtained using three different techniques

\begin{tabular}{lccc}
\hline $\boldsymbol{\beta}-\mathbf{M n O}_{\mathbf{2}}$ (P42/mnm) & $\mathbf{a}=\mathbf{b}(\mathbf{\AA})$ & $\mathbf{c}(\AA)$ & $\mathbf{V}(\AA \mathbf{3})$ \\
\hline DFTB & 4.405 & 2.870 & 55.70 \\
EXP (Barudzija et al., 2016) & 4.408 & 2.871 & 55.81 \\
DFT ( Rogers., 1993) & 4.414 & 2.860 & 56.44 \\
\hline
\end{tabular}

Table 3: Coordinates/position of atoms in the bulk structure of $\beta-\mathrm{MnO}_{2}$

\begin{tabular}{lcccccc}
\hline & \multicolumn{2}{c}{ DFTB } & \multicolumn{2}{c}{$\begin{array}{c}\text { EXP (Barudzija } \\
\text { et al.,2016) }\end{array}$} & \multicolumn{2}{c}{$\begin{array}{c}\text { DFT (Rogers., } \\
\text { 1993) }\end{array}$} \\
\hline Site & $\mathrm{Mn}$ & $\mathrm{O} 1$ & $\mathrm{Mn}$ & $\mathrm{O} 1$ & $\mathrm{Mn} 1$ & $\mathrm{O} 1$ \\
\hline $\begin{array}{l}\text { Wyckoff } \\
\text { position }\end{array}$ & $2 \mathrm{a}$ & $4 \mathrm{f}$ & $2 \mathrm{a}$ & $4 \mathrm{f}$ & $2 \mathrm{a}$ & $4 \mathrm{f}$ \\
$\mathrm{X}$ & 0.0 & 0.2 & 0.0 & 0.3 & 0.0 & 0.2 \\
$\mathrm{Y}$ & 0.0 & 0.2 & 0.0 & 0.3 & 0.0 & 0.0 \\
$\mathrm{Z}$ & 0.0 & 0.0 & 0.0 & 0.0 & 0.0 & 0.0 \\
Occupancy & 1.0 & 1.0 & 1.0 & 1.0 & 1.0 & 1.0 \\
\hline
\end{tabular}




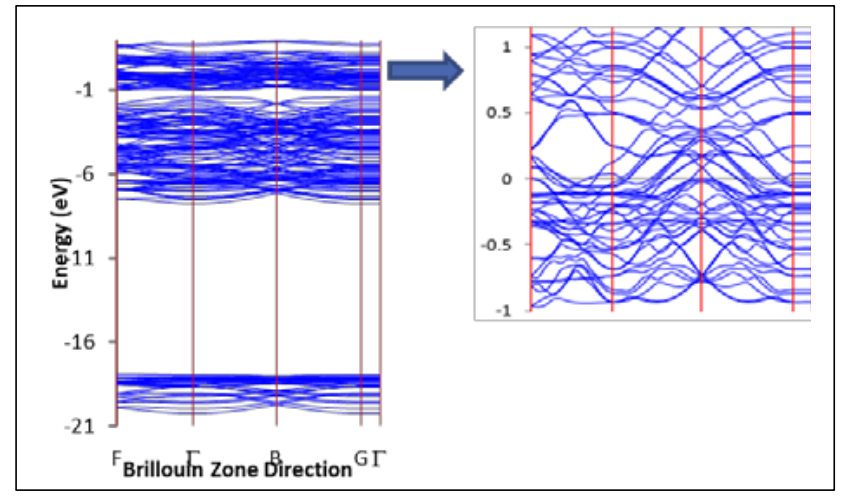

Figure 2: Electronic band structures of $\beta-\mathrm{MnO}_{2}$ using DFT

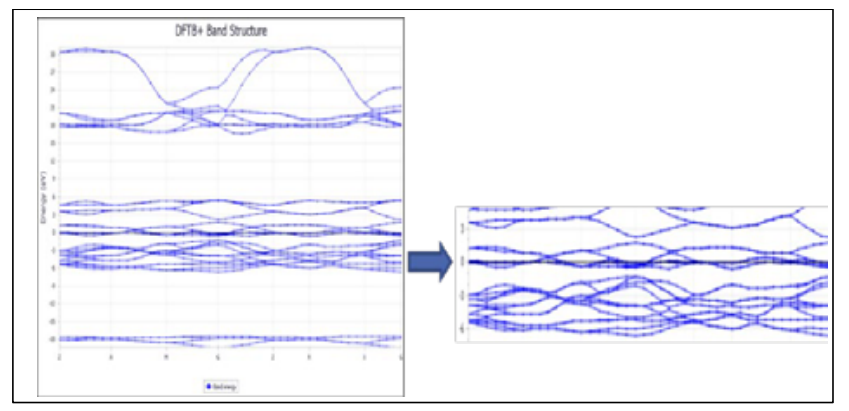

Figure 3: Electronic band structures of $\beta-\mathrm{MnO}_{2}$ using DFTB

occupation and a zero DOS means that no states are available for occupation.

\section{Band structures}

The calculated band structures for $\mathrm{MnO}_{2}$ display the overlap of orbitals at the fermi level from both the DFT and DFTB codes. However, the absence of an electronic band indicates that both structures are metallic. In metal, the highest energy band is reasonably packed or the highest packed band and the following vacant band overlaps energy.

\section{Density of states (DOS)}

Figure 4 below shows the calculated total and partial electronic density of states (TDOS/PDOS) for $\mathrm{MnO}_{2}$ using DFT and DFTB techniques. However, fig 4 (a) shows the DOS from DFT signifies how all the atoms contributed to each band. The Manganese (Mn) PDOS is dominated by the $\mathrm{s}$ and $\mathrm{p}$ orbitals with two broad peaks at -85 to $-78 \mathrm{eV}$ and -55 to $-46 \mathrm{eV}$, respectively at the conduction band. The $\mathrm{d}$ orbital contributes to the conduction band at around -9 to $0 \mathrm{eV}$. And further, the $\mathrm{d}$ orbital of $\mathrm{Mn}$ contributes to the valence band from 0 to $+19 \mathrm{eV}$.

The is no contribution of the $\mathrm{s}$ and $\mathrm{p}$ orbitals at the valence band. The oxygen atom shows the contribution of $\mathrm{s}$ and $\mathrm{p}$ orbital at the conduction band from -20 to $-17 \mathrm{eV}$ and -9 to $0 \mathrm{eV}$, respectively. The $\mathrm{p}$ orbital contributes to the valence band at 0 to $+8 \mathrm{eV}$. Additionally, the $\mathrm{s}$ and $\mathrm{d}$ orbital does not show any contribution at the valence band.

Figure 4 (b) shows the DOS from DFTB findings signifying how each one of the orbitals contributed to each band. The conduction band of the Mn atom exhibits one peak of d orbital between -8 and $0 \mathrm{eV}$. The is no sign of the $\mathrm{s}$ and $\mathrm{p}$ orbital of $\mathrm{Mn}$ at the conduction

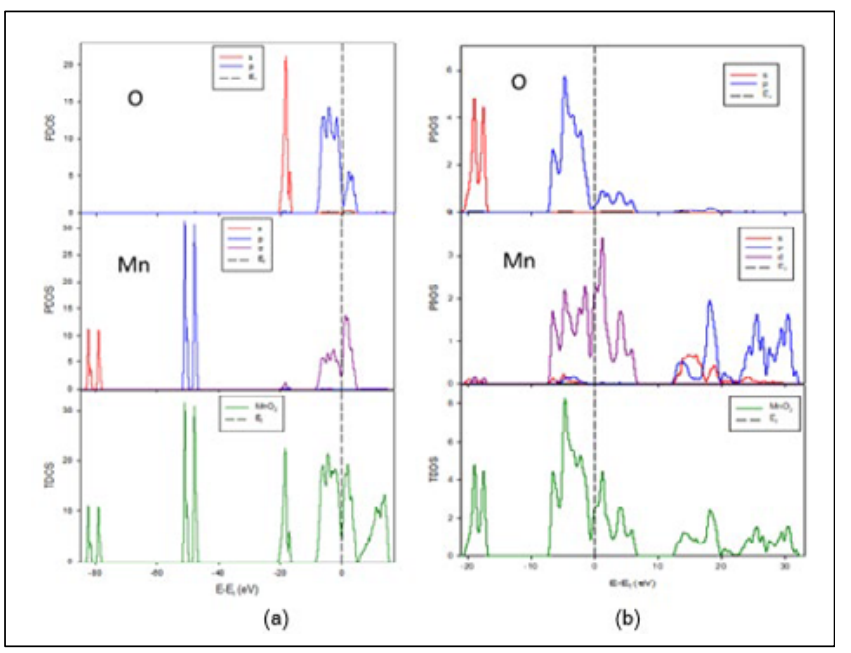

Figure 4: Comparison of the DOS for $\mathrm{MnO}_{2}$ from (a) DFT and (b) DFTB results

band. From the valence band the s, p, and d orbital contribute to the band at 11 to $+22 \mathrm{eV},+11$ to $+33 \mathrm{eV}$, and 0 to $+8 \mathrm{eV}$, respectively. Furthermore, the PDOS of the $\mathrm{O}$ atom at conduction band displays the two peaks of $\mathrm{s}$ and $\mathrm{p}$ orbital from -21 to $-17 \mathrm{eV}$ and -8 to $-1 \mathrm{eV}$, respectively. The $\mathrm{d}$ orbital does not exist for the $\mathrm{O}$ atom.

However, there is a slight shift of the peaks from all the orbitals from the conduction to the valence band. A slight shift is also noticed from the TDOS when both techniques are employed. The DFTB technique might be the reason behind the shift of the TDOS because it usually deals with systems of numerous atoms. The electronic band structures and the total density of states using both the DFT and DFTB techniques are in good agreement with one another since there is an overlap of orbitals at the Fermi level and the absence of a gap at the Fermi level which confirms that the $\mathrm{MnO}_{2}$ system is metallic. Previous experimental work (Xiong et al.,2013) has shown similar results to DFT and DFTB obtained.

\section{Conclusion}

We successfully developed $\beta-\mathrm{MnO}_{2}$ parameters from DFTB, for which the lattice parameters obtained were comparable with the lattice parameters from experiments and DFT within the percentage difference of $2 \%$. We can confidently say that the structural and electronic properties of $\beta-\mathrm{MnO}_{2}$ have been explored effectively. The contribution of all the orbitals of the atoms was properly discussed, the electronic band structures and the total density of states shown good agreement when using both the DFT and DFTB techniques. However, the absence of a bandgap at the Fermi level from both the electronic band structures and total density of states implies the metallic nature of the $\beta-\mathrm{MnO}_{2}$ system. The results help to understand the intrinsic structural and electronic properties of the phenomenon observed but these findings are important in preserving the crystal structure $\beta-\mathrm{MnO}_{2}$.

\section{Acknowledgments}

The authors recognize for their financial support. The calculations were carried out utilizing computer assets at the Material Modelling Centre (MMC) at the University of Limpopo and the Centre for High-Performance Computing (CHPC) based in Cape Town. 


\section{References}

1. Delley B, 1990. An all-electron numerical method for solving the local density functional for polyatomic molecules. J. Chem. Phys, Volume 92, pp. 508-517.

2. Zachau-Christiansen B, West K, Jacobsen T, and Skaarup S, 1994. Oxidation behaviour of mechanically activated Mn3O4 by TGA/DSC/ XRPD. Solid State Ionics, Volume 70-71, p. 401.

3. Kim C.-H, Akase Z, Zhang L, Heuer A.H, Newman A.E, and Hughes P.J, 2006. The structure and ordering of $\varepsilon-\mathrm{MnO} 2$. J. Solid State Chem., Volume 179, pp. 753-774.

4. Balachandran D, Morgan D and Ceder G, 2002. J. Solid State Chem, Volume 166, p. 91

5. Cockayne E, and Li L, 2012. A first-principles investigation of $\alpha, \beta$, and $\gamma-\mathrm{MnO} 2$ as potential cathode materials in Al-ion batteries. Chem. Phys. Lett, Volume 544, pp. 53-58.

6. Xiong G, Hembram K.P.S.S, Reifenberger R.G, and Fisher T.S, 2013. $\mathrm{MnO} 2$-coated graphitic petals for supercapacitor electrodes. Journal of Power Sources, Volume 227, pp. 254-259.

7. Goodenough J.B, Thackeray M.M, David W.I.F. and Bruce P.G, 1984. Rev. Chim. Miner., Volume 21, p. 435.

8. Hunter J.C, 1981. Preparation of a new crystal form of manganese dioxide: $\lambda$-MnO2. J. Solid State Chem., Volume 39, p. 142

9. Perdew J.P, Burke K, and Ernzwerhof M, 1996. First Principles Study of Oxygen Adsorption on Li-MO2 (M = Mn, Ti and V) (110) Surface. Phys. Rev, Volume 77
10. Dahn J.R, Von Sacken U, Juzkow M. W, and Al-Janaby H, 1991. Rechargeable LiNiO2/Carbon Cells. J. Electrochem. Soc., Volume 138, pp. 2207-2208.

11. Rogers K.D, 1993. An X-ray diffraction study of semiconductor and metallic vanadium dioxide. Powder Diffr, Volume 8, p. 240.

12. MacLean L.A.H, and Tyle F.L, 1996. The Structure of Fully H-Inserted $\gamma$-Manganese Dioxide Compounds. J. Solid State Chem, Volume 123, p. 150.

13. Thackeray M.M, De Kock A, De Picciotto L.A, and Pistoia G, 1989. Synthesis and characterization of $\gamma-\mathrm{MnO} 2$ from LiMn2O4. J. Power Sources, Volume 26, p. 355

14. Thackeray M.M, David W.I.F., Bruce P.G, and Goodenough J.B 1983. Exploratory synthesis of new Iron(ii,iii)-containing polyanion framework solids of electrochemical and magnetic interest. Mater. Res. Bull, Volume 18, pp. 461-468.

15. Mo S.D. and Ching W.Y, 1995. International Journal of Engineering and Advanced Technology. Phys. Rev., Volume 51, pp. 1302-1306.

16. Barudzija T, Kusigerski V, Cvjeticanin V, Sorgic V, Perovic M, and Mitric M, 2016. Structural and magnetic properties of hydrothermally synthesized $\beta-\mathrm{MnO} 2$ and $\alpha-\mathrm{KxMnO} 2$ nanorods. Journal of alloys and compounds, Volume 665, pp. 261-270.

17. Barudzija T, Kusigerski V, Cvjeticanin N, Sorgic S, Perovic M, and Mitric M, 2016. Structural and magnetic properties of hydrothermally synthesized $\beta-\mathrm{MnO} 2$ and $\alpha-\mathrm{KxMnO} 2$ nanorods. Journal of alloys and compounds, Volume 665, pp. 261-270. 